\title{
Combined Supervised / Unsupervised Algorithm for Skin Detection: A Preliminary Phase for Face Detection
}

\author{
Eyal Braunstain and Isak Gath \\ Department of Biomedical Engineering \\ Technion - Israel Institute of Technology, Haifa 32000, Israel \\ seyalbra@tx.technion.ac.il, isak@bm.technion.ac.il
}

\begin{abstract}
Skin detection in color images is of great importance for the computer vision research community. Many existing skin detection algorithms are characterized by high false detection rates. The proposed algorithm performs offline learning of skin color, but also of "false-skin colors", which may be misclassified as skin using regular histogram or Gaussian skin color models. This supervised learning of false-skin colors produces a significant reduction in false detection rates. Our aim is to extract skin blobs that are suspected to contain faces, which are usually ellipsoid-shaped.

Thus, to extract these blobs, an unsupervised optimal fuzzy clustering (UOFC) algorithm is applied in the spatial image space. Blobs segmented by the clustering procedure are then examined by specific features, e.g. geometrical, to classify them as face candidates.

Sample runs of the algorithm on a bank of images show high skin detection rates with reduced false detection rates.
\end{abstract}

Keywords: Skin Detection, Supervised Learning, Unsupervised Learning, Optimal Fuzzy Clustering, Likelihood Estimation.

\section{Introduction}

Skin color serves as a powerful cue for people detection in unconstrained environments, with applications ranging from detection and recognition of faces, of limbs gestures and movements for human-computer interaction systems, of naked people on the WWW etc. Skin color can be modeled in various color spaces (e.g. RGB, YCbCr, YES) and by various models, e.g. single Gaussian, mixture of Gaussians, or by histograms. The classification of a test-pixel can be made by various methods, e.g. by a probability threshold decision-making. Many skin detection techniques produce good detection rates, but may result in relatively high false detection rates [11].

The performance of histogram models was found to be superior to Gaussian mixture models, both in accuracy and in computational cost [3].

Much of previous research work on skin classification used the mixture of Gaussians model, comprised of a weighted sum of Gaussian kernels. The advantage of the model lies mainly in its ability to generalize well on a small training data set [3], [5]. 
The authors of [6] suggest that normalization of RGB color values by the sum $(\mathrm{R}+\mathrm{G}+\mathrm{B})$ yields a color space that is more efficient for skin color segmentation, since the sensitivity to variation of skin color is reduced. This is of high importance under variable illumination conditions. In [10], the skin color histograms of nine color spaces are examined, and it is stated that the distribution in r-g (Normalized R-G) space is compatible with a unimodal elliptical Gaussian model.

The authors of [1] suggest an image annotation scheme, based on supervised classification of colors, and learning classes' thresholds from ROC curves. In the present work, we suggest concomitant modeling of skin color and false-skin colors, i.e. colors that might be misclassified as skin by a skin color classifier, but do not actually represent skin.

The Unsupervised Optimal Fuzzy Clustering (UOFC) algorithm, developed by Gath and Geva [2], was tested for various applications, e.g. clustering of EEG signal recordings, in order to classify the signal into various sleep stages. In this paper, this algorithm was utilized to detect skin blobs that were suspected to contain faces.

The work is focused on white and asian skin-colored people. Dealing with more variable skin colors may require the construction of additional skin colors histograms for the different trained skin classes.

\section{Skin Blobs Detection as Preliminary Phase to Face Detection}

\subsection{Training of the Skin Color and False-Skin Colors Classifiers}

In the suggested algorithm, color is represented in CIE Lab space, which is perceptually more uniform than many other color spaces, e.g. RGB, HSV [7]. This enables the use of uniform color metric for color-related decision-making (e.g. clustering). For skin detection purposes, only the chroma components $(a, b)$ are used, and luminance $(\mathrm{L})$ is disposed. This is done since the intensity of the reflected light from skin of different people, or in variable lighting conditions, varies considerably, while the chroma remains relatively constant [7].

First, a single skin classifier was trained by manually labeling skin pixels and constructing a 2-D color histogram in CIE-Lab space, using a-b chroma values only.

We propose a supervised learning approach of skin color, as well as false-skin color, i.e. color values that allegedly may be classified as skin by a simple histogram classifier, but do not belong to skin. The suggested approach is aimed at lowering the false detection rate in skin classification. A skin histogram is constructed from a training set of 20 skin hand-labeled pictures. This histogram is used to classify skin on a validation set of 20 different images, in which it was observed that most false detection pixels belonged to 3 prime colors - brown, red and white. The training and validation sets are considered of appropriate sizes to avoid overfitting to the learning data. 
The false detection pixels observed in the validation set were manually labeled to three new distinct training sets for brown, red and white, and three new histograms in CIE-Lab space (chroma components only, a-b) for these colors were constructed.

These histograms were smoothed by a Gaussian function; see fig. (1). It can be observed that there exists a significant overlap between different histograms, mainly between the skin to the brown and red histograms.

To train parameters for class $\mathrm{W}_{\mathrm{i}}$, let us assume that each color class can be represented by a single-Gaussian model.

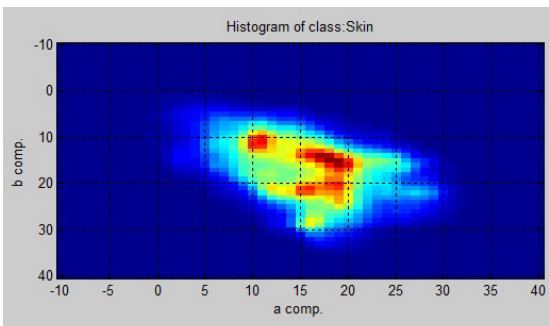

Histogram of class:Red

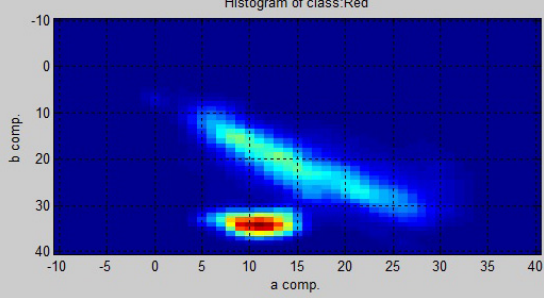

Histogram of class:Brown

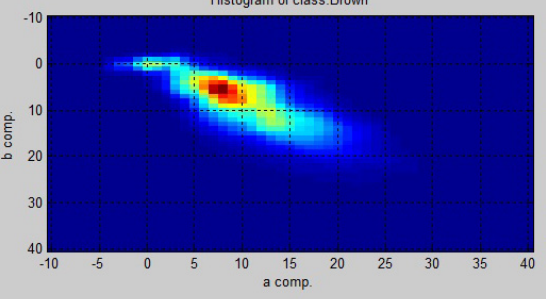

Histogram of class:White

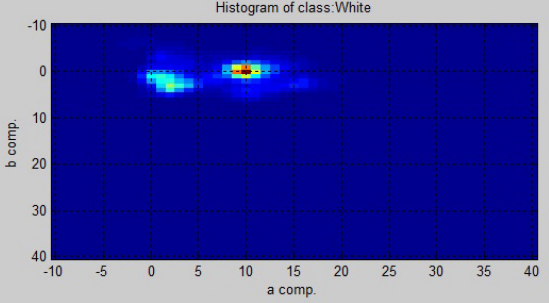

Fig. 1. Histograms of color classes in CIE-Lab space, by a-b chroma components. Upper left- skin color; upper right- brown; lower left- red; lower right- white.

This assumption is somewhat inaccurate, since certain color distributions are not unimodal; however, the discussed histograms display a distribution around a primary mode in $a-b$ chroma space. Chroma values $(a, b)$ are distributed in the range $[-110$, $110]$, and in fig. (1) are displayed in partial range [-10, 40].

Given the hand-labeled pixels of color class $W_{i}$, the mean chroma vector $m_{i}$ and chroma covariance matrix $\mathrm{K}_{\mathrm{i}}$, for class $\mathrm{W}_{\mathrm{i}}$ are:

$$
m_{i}=\left[m_{a}, m_{b}\right]^{T} \quad K_{i}=\left[\begin{array}{cc}
\sigma_{a}^{2} & \sigma_{a b} \\
\sigma_{a b} & \sigma_{b}^{2}
\end{array}\right]
$$

Given a vector of chroma values for some pixel $\mathrm{p}, \mathrm{x}_{\mathrm{p}}=\left[\mathrm{a}_{\mathrm{p}}, \mathrm{b}_{\mathrm{p}}\right]$, a unimodal Gaussian distribution is fitted to class $\mathrm{W}_{\mathrm{i}}[1]$ :

$$
p\left(x_{p} \mid W_{i}\right)=(2 \pi)^{-1}\left|K_{i}\right|^{-\frac{1}{2}} \exp \left\{-\frac{1}{2} \lambda_{p}^{i}\right\}
$$


Where $\lambda_{p}^{i}$ is defined in Eq. (3). The contours of the Normal pdf of each class define an ellipse, centered at $\mathrm{m}_{\mathrm{i}}$, and whose principal axes are determined by $\mathrm{K}_{\mathrm{i}}$.

$$
\lambda_{p}^{i}=\left[x_{p}-m_{i}\right]^{T} K_{i}^{-1}\left[x_{p}-m_{i}\right]
$$

Given $\mathrm{N}$ color classes, $\mathrm{N}$ scalar values $\lambda_{p}^{i}$ are to be calculated for pixel $\mathrm{p}$, which are proportional to the probability that the pixel belongs to class $\mathrm{W}_{\mathrm{i}}$.

A specific threshold was learned for each class $\mathrm{W}_{\mathrm{i}}$, denoted $\mathrm{t}^{\mathrm{i}}$, by an optimum true positive (TP) - false positive (FP) analysis using the training set data. An ROC curve is generated for each color class $\mathrm{i}$, plotting the TP vs. FP rates for a variety of threshold values $\lambda_{t}[1]$.

The TP and FP rates for a class are calculated over the training set of that class. For pixel p, with some chosen threshold $\lambda_{t}$, we have the TP and FP rates for that pixel:

$$
\begin{aligned}
t p_{p}^{\lambda_{t}, I_{j}^{i}} & =\left\{\begin{array}{ll}
1 & \text { if } p \text { labeled } W_{i} \& \lambda_{p}^{i} \leq \lambda_{t} \\
0 & \text { otherwise }
\end{array}\right\} \\
f p_{p}^{\lambda_{t}, I_{j}^{i}} & =\left\{\begin{array}{ll}
1 & \text { if } p \text { not labeled } W_{i} \& \lambda_{p}^{i} \leq \lambda_{t} \\
0 & \text { otherwise }
\end{array}\right\}
\end{aligned}
$$

Where $I_{j}^{i}$ denotes image $\mathrm{j}$ in training set, with pixels labeled as class $\mathrm{i}$.

From the pixels' true positive and false positive indicators defined in Eq. (4), we deduce by image summation the TP and FP rates for class $i$, and threshold $\lambda_{t}$. The TP, FP rates are calculated for various threshold values, thus obtaining an ROC curve. The optimum threshold for class $\mathrm{i}, t^{i}$, is computed as the intersection point of the ROC curve and the line $T P=1-F P$, [6]. This threshold simultaneously maximizes TP and minimizes FP, which serves the motivation of minimizing the false detection rate in skin classification.

\subsection{Skin Color Classification}

The classification of pixel $\mathrm{p}$ in a test image is performed by the application of $\mathrm{N}$ binary hypothesis tests based on Eq. (3), with thresholds $\mathrm{t}^{\mathrm{i}}$ for each color class:

$$
c_{p}^{i}=\left\{\begin{array}{lll}
1 & \lambda_{p}^{i} \leq t^{i} & \left\{p \in W_{i}\right\} \\
0 & \lambda_{p}^{i}>t^{i} & \left\{p \in \bar{W}_{i}\right\}
\end{array}\right\} i=\{1, . ., N\}
$$

$c_{p}^{i}$ is a binary classification of pixel $\mathrm{p}$ to class $\mathrm{W}_{\mathrm{i}}$, equal to 1 if $\mathrm{p}$ is in this color class.

This is followed by an MAP (Maximum a posteriori) decision rule, for pixels which are found to belong to more than one color class according to hypotheses. 
Each pixel is classified to one of the 4 color classes, or to the "other" class ("rest of the world"), whose distribution is not modeled. The MAP absolute decision rule is defined by:

$$
\text { pixel } p \in W_{k} \text { iff } \operatorname{Pr}\left(W_{k} \mid x_{p}\right)=\max \left\{\operatorname{Pr}\left(W_{i} \mid x_{p}\right)\right\}_{i=1 . . N}
$$

Where, $\operatorname{Pr}\left(W_{i} \mid x_{p}\right)$ is attained by Bayes' theorem.

Finally, after the classification of the pixels to color classes for the test image, the pixels that were classified to the skin color class were extracted.

\subsection{Extracting Skin Blobs Suspected as Faces by Optimal Fuzzy Clustering}

The goal of the algorithm is to extract from a test image the skin blobs, which probably contain faces. Faces are often grossly described as elliptic-shaped, so we would like to extract blobs of ellipsoidal shape from the skin image produced so far. No prior knowledge of the number of faces in the image exists. A modified version of the Unsupervised Optimal Fuzzy Clustering algorithm was utilized. This algorithm, combining the fuzzy K-means and fuzzy maximum likelihood estimation (FMLE) has been shown to perform well in cases of clusters of variable hyper-ellipsoidal shapes. The data to be clustered is the spatial image points ( $\mathrm{x}, \mathrm{y}$ coordinates of the image). To reduce the computational load of the clustering algorithm, the skin points in the spatial image domain have been sub-sampled by a sampling grid. However, this required interpolation of the clustered data back to the original image grid. Let us denote by $\mathrm{X}$ the data matrix of skin image points $(x, y)$, i.e. feature vectors, and assume grossly that the initial number of clusters is the number of adjoined components in the skin image. The number of ellipsoidal face aggregates does not necessarily correlate with the number of adjoined components, but considering the existence of noisy components in the image, it was found to usually lead to accurate clustering results. The UOFC algorithm has been applied to the features matrix, X. In its first stage, the fuzzy K-means algorithm was applied, minimizing the following objective function, with respect to $\mathrm{U}$, the fuzzy membership matrix, and $\mathrm{V}$, the prototypes - clusters centroids:

$$
J_{q}(U, V)=\sum_{j=1}^{N} \sum_{i=1}^{K}\left(u_{i j}\right)^{q} d^{2}\left(X_{j}, V_{i}\right) \quad K \leq N
$$

where $\mathrm{N}$ is the number of data points, $\mathrm{K}$ is the number of prototypes (assumed smaller than $\mathrm{N}$ ), q, the weighting exponent for the fuzzy memberships, controls the "fuzziness" of the resulting clusters [8]. $\mathrm{d}$ is a defined metric (e.g. Euclidean).

Fuzzy K-means is run through an iterative optimization procedure. The end results of the fuzzy K-means clustering, $\mathrm{V}$ and $\mathrm{U}$, serve as initial conditions to the FMLE algorithm, whose stages are given by: 
1) Initial clusters centroids are given by the end results of fuzzy K-means stage.

2) To address the problem of clustering data that is not necessarily spherically distributed, a $2^{\text {nd }}$ stage, based on maximum likelihood estimation (MLE) is carried out, with an "exponential" distance $d_{e}$ [2]. It will be used to compute $\mathrm{h}\left(\mathrm{i} \mid \mathrm{X}_{\mathrm{j}}\right)$, the posterior probability of selecting cluster $\mathrm{i}$, given feature vector $\mathrm{X}_{\mathrm{j}} \mathrm{h}\left(\mathrm{i} \mid \mathrm{X}_{\mathrm{j}}\right)$ and the exponential distance $\mathrm{d}_{\mathrm{e}}$ are given by:

$$
\begin{aligned}
& h\left(i \mid X_{j}\right)=\frac{\frac{1}{d_{e}^{2}\left(X_{j}, V_{i}\right)}}{\sum_{k=1}^{K} \frac{1}{d_{e}^{2}\left(X_{j}, V_{k}\right)}} \\
& d_{e}^{2}\left(X_{j}, V_{i}\right)=\frac{\left[\operatorname{det}\left(F_{i}\right)\right]^{1 / 2}}{P_{i}} \exp \left\{\left(X_{j}-V_{i}\right)^{T} F_{i}^{-1}\left(X_{j}-V_{i}\right) / 2\right\}
\end{aligned}
$$

At the first FMLE iteration, the posterior probabilities $h\left(i \mid X_{j}\right)$ can be inferred from the memberships $U$. To calculate $d_{e}, F_{i}$ (the fuzzy covariance matrix of cluster i) and $\mathrm{P}_{\mathrm{i}}$ (the a-priori probability) need to be computed:

$$
F_{i}=\frac{\sum_{j=1}^{N} h\left(i \mid X_{j}\right)\left(X_{j}-V_{i}\right)\left(X_{j}-V_{i}\right)^{T}}{\sum_{j=1}^{N} h\left(i \mid X_{j}\right)}, \quad P_{i}=\frac{1}{N} \sum_{j=1}^{N} h\left(i \mid X_{j}\right)
$$

3) Update clusters centroids by Eq. (11), and posterior probabilities by Eq. (8).

$$
\widehat{V}_{i}=\frac{\sum_{j=1}^{N}\left(h_{i j}\right)^{2} X_{j}}{\sum_{j=1}^{N}\left(h_{i j}\right)^{2}}
$$

4) For a predefined threshold $\mathcal{E}$, if the following condition holds, than stop: $\max _{i j}\left\{\left|h_{i j_{-} \text {new }}-h_{i j}\right|\right\}<\varepsilon$. Otherwise, go to step 3, and iterate steps 3, 4 until convergence.

The end results of the UOFC algorithm are $\mathrm{H}$, the posterior probabilities matrix (equivalent to the fuzzy membership matrix, $\mathrm{U}$ ), and $\mathrm{V}$, the prototype centroids. To determine, for each pixel, to which cluster it belongs, we utilize a hard-partition rule on the clusters' probabilities of $\mathrm{H}$ - each pixel will be attributed to the cluster to which its posterior probability is maximal. The end result is an absolute partition (segmentation) of the image. It is possible for pixels belonging to a specific cluster to be spatially disjoined, e.g. as two groups of pixels that are very close to each other in image 
space. However, disjoined pixels are to be regarded as separate clusters, so an analysis on the clusters map was performed, to separate disjoined components into different clusters. This might increase the number of clusters, but produces a more natural segmentation of the image. However, somewhat on the contrary, under certain geometrical conditions, neighboring clusters which are spatially adjoined in image space can be merged into one cluster.

\subsection{Post-processing of Skin Blobs}

Skin segments (clusters) have been extracted from the image. The post-processing of these skin blobs is comprised of morphological operations and geometrical tests. The validity of each cluster's fitting ellipse main axes ratio and cluster bounding box $\mathrm{X}-\mathrm{Y}$ sizes ratio are checked to converge to pre-determined thresholds. Skin blobs can also be filtered by disposing of regions with high-amplitude variation in intensity [4]. In this work, a lower threshold is applied on the color variance of skin clusters, to filter skin segments, assuming that a face area is generally characterized by larger color variance than most other skin areas (e.g. hands, legs) due to the color variability of mouth, eyes and possibly facial hair, against skin color. This phase of post-processing enables further reduction of the number of detected skin blobs suspected as faces.

\section{Skin Blobs Detection Experimental Results}

The algorithm was tested on a bank of 50 self-picked images, mostly from the WWW, of white and Asian people, with variable background and lighting conditions.

A standard faces database was not used (e.g. Caltech faces database [12]), since the collected images were chosen to contain also skin parts other than faces, with indoor and outdoor variable backgrounds. Images were chosen to contain 2-6 individuals.

Sample results of the algorithm on an outdoor image of a group of 6 Asian people are presented (Fig. 2), with a distinct variability in visible skin color - from pale to brownish hue. The unified algorithm serves as more than a skin-pixels classifier, but is not a face detector. It would be wrong to define skin detection rates by simply counting skin pixels, since many skin patches (e.g. hands, legs) are deliberately filtered out. The percentage of undeleted faces on the test bank was $\sim 97 \%$. The false detection (FP) rate is intuitively related to pixels falsely detected as skin, and the average rate was $7 \%$. The skin FN measure is high, since non-face skin patches were deleted. Thus, TP rate for skin may be low, but is not a valid performance measure. Table 1 provides a comparison to other skin detection techniques.

Fig. (2a) is the test image. Fig. (2b) is the skin classification image when training is performed on skin color class alone. Fig. (2c) is the skin classification image when the training is performed on skin class and false detection classes (false skin classes). It is apparent that the false detections existing in (2b) - some white background patches and brown hair of the figures in the image, are now diminished. 
a

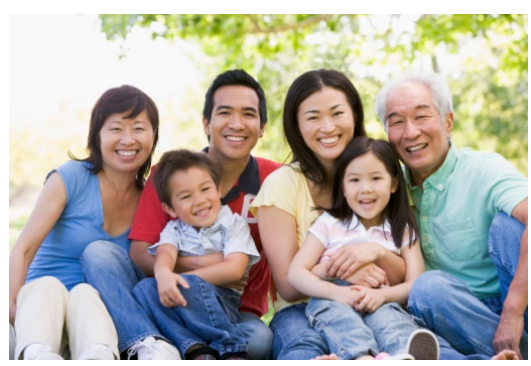

$\mathrm{c}$

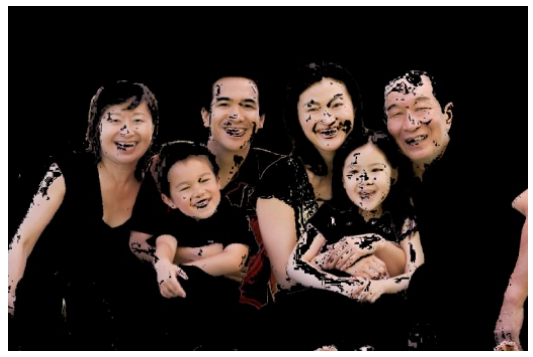

e

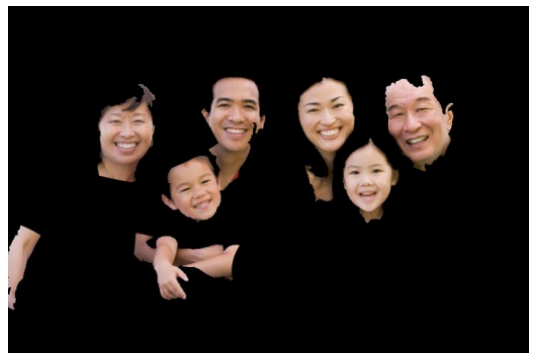

b

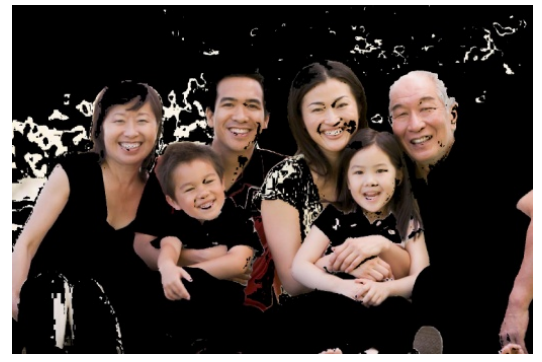

d

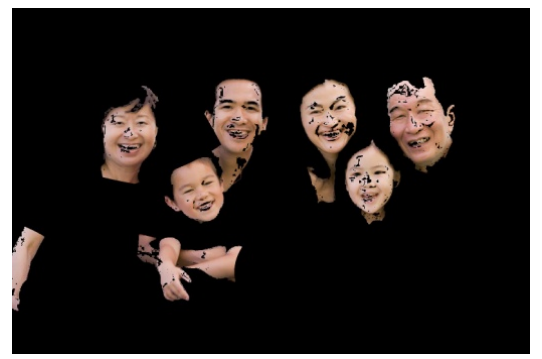

f

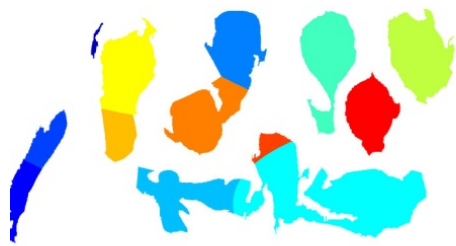

Fig. 2. Skin detection algorithm results. (a) Test image for skin blobs detection.

(b) Skin pixels classification; training of skin class only (apparent false detections).

(c) Skin pixels classification; skin class and false skin classes (red, brown and white).

(d) Running Optimal fuzzy clustering algorithm over image in (c), with post processing.

(e) Morphological filling holes in (d). The produced image can be a fed to a face detector.

(f) An RGB labeling map of the clusters computed by FMLE clustering.

Table 1. Comparison of TP and FP measures with other skin detection techniques

\begin{tabular}{|c|c|c|}
\hline Method & Skin TP (\%) & Skin FP (\%) \\
\hline Our algorithm & $\begin{array}{c}\text { Not valid (See page 7) } \\
\text { (Face detection rate - 97) }\end{array}$ & 7 \\
\hline Jones \& Rehg [3] & 93.9 & 8 \\
\hline Maximum Entropy Model [13] & 80 & 8 \\
\hline Wu \& Ai [14] & 92.9 & NA \\
\hline
\end{tabular}


The classification of (2c) caused the increase in miss-detection of some skin pixels, but the effect is somewhat negligible, and is mostly corrected by morphological operations performed at a later stage. Fig. (2d) presents the result of running Optimal Fuzzy Clustering algorithm on image in (2c), with post processing, thus erasing skin blobs that probably do not contain a face - hands and chest skin blobs in this case. This is done by some threshold criteria, e.g. $\mathrm{x}$ and $\mathrm{y}$ axes components ratio in bounding box, lower threshold on extent of cluster blob pixels to full cluster bounding box, lower threshold on standard deviation of cluster color values, etc.

Fig. (2e) presents the result of morphological filling of holes of the image in (2d), thus including eyes and mouth of the figures in the image. This image is comprised of skin blobs that are suspected to contain faces. It can be observed that besides some hands blobs, this mask image contains mostly "floating heads", which is very much desirable. Utilizing a face detection or recognition algorithm on this filtered skin image will require scanning of much less area than the full image. Fig. (2f) demonstrates the RGB labeling map of the clusters computed by Optimal FMLE clustering. Each color designates a different cluster in spatial image space. The advantage of FMLE clustering (segmentation) is apparent, especially in the middle of the image, in the area of the adult male and young child below him; we can clearly see in the clustering map that the UOFC algorithm succeeded in segmenting and distinguishing the two different heads of the adult and child, which are both ellipsoidal in shape and are adjoined components in the skin mask image. The algorithm was shown to be efficient on variable backgrounds and different shapes.

\section{$4 \quad$ Summary and Conclusions}

In the presented study, a new algorithm is described for the detection of skin blobs in color images, suspected to contain faces. Supervised learning of skin color histogram was performed, and was tested on a validation set. From the classification results on that set, three major false-skin color classes were recognized - red, brown and white, and thus three additional histograms were constructed, to model them. A Gaussian model was fitted to each class. Given a new test image, $\mathrm{N}$ hypothesis tests (for $\mathrm{N}$ classes) were implemented, followed by an MAP decision rule, to allocate a specific color class to each pixel. Pixels in the image that belonged to the skin class were then extracted. This skin mask image was segmented by a modified UOFC algorithm, followed by morphological and geometrical post-processing performed on the segmentation result, to yield the final result of filtered skin blobs image.

The algorithm was tested on an image bank containing 50 images, of variable background with different shapes, and variable lighting conditions. It produced very good detection performance, with reduced false detection rates.

The combination of supervised learning of skin color and false-skin colors models, in addition to the utilization of the UOFC algorithm to extract grossly ellipsoidal skin blobs that might contain faces, creates a skin mask that is drastically relieved of irrelevant segments, while preserving the blobs in the image that contain faces. 
The suggested algorithm can serve as a preliminary phase to a face detection or recognition algorithm, by reducing significantly the image area that has to be scanned in the image to detect or recognize faces.

\section{References}

1. Saber, E., Murat Tekalp, A., Eschbach, R., Knox, K.: Automatic Image Annotation Using Adaptive Color Classification. Graphical Models and Image Processing 58(2), 115-126 (1996)

2. Gath, I., Geva, A.B.: Unsupervised Optimal Fuzzy Clustering. IEEE Transactions on Pattern Analysis and Machine Intelligence 11(7) (1989)

3. Jones, M.J., Rehg, J.M.: Statistical Color Models with Application to Skin Detection. International Journal of Computer Vision 46(1), 81-96 (2002)

4. Forsyth, D.A., Fleck, M., Bregler, C.: Finding naked people. In: Buxton, B.F., Cipolla, R. (eds.) ECCV 1996. LNCS, vol. 1065, Springer, Heidelberg (1996)

5. Schiele, B., Waibel, A.: Gaze tracking based on face-color. In: Proceedings of the International Workshop on Automatic Face- and Gesture-Recognition, Zurich, Switzerland, pp. 344-349 (1995)

6. Terrillon, J.-C., David, M., Akamatsu, S.: Automatic Detection of Human Faces in Natural Scene Images by Use of a Skin Color Model and of Invariant Moments. In: Proceedings of Third IEEE International Conference on Automatic Face and Gesture Recognition FG (1998)

7. Cai, J., Goshtasby, A.: Detecting human faces in color images. Image and Vision Computing 18, 63-75 (1999)

8. Bezdek, J.C.: Pattern Recognition with Fuzzy Objective Function Algorithms, New York, Plenum (1981)

9. Bezdek, J.C.: Fuzzy Mathematics in Pattern Classification. Ph.D. dissertation, Cornell Univ., NY (1973)

10. Terrillon, J.-C., Akamatsu, S.: Comparative Performance of Different Chrominance Spaces for Color Segmentation and Detection of Human Faces in Complex Scene Images. Vision Interface, Trois-Rivières, Canada (1999)

11. Vezhnevets, V., Sazonov, V., Andreeva, A.: A Survey on Pixel-Based Skin Color Detection Techniques. In: Proc. Graphicon (2003)

12. Caltech face database (1999), http://www.vision.caltech.edu/html-files/archive.html

13. Jedynak, B., Zheng, H., Daoudi, M., Barret, D.: Maximum entropy models for skin detection. Tech. report, Universite des Sciences et Technologies de Lille, France (2002)

14. Wu, Y.-W., Ai, X.-Y.: Face Detection in Color Images Using AdaBoost Algorithm Based on Skin Color Information. Knowledge Discovery and Data Mining (2008) 